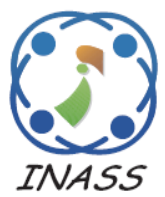

http://www.inass.org/

\title{
MLBO: Mixed Leader Based Optimizer for Solving Optimization Problems
}

\author{
Fatemeh Ahmadi Zeidabadi ${ }^{1}$ \\ Mohammad Dehghani ${ }^{2 *}$ \\ Sajjad Amiri Doumari ${ }^{1}$ \\ Om Parkash Malik ${ }^{3}$ \\ ${ }^{I}$ Department of Mathematics and Computer Sciences, Sirjan University of Technology, Sirjan, Iran \\ ${ }^{2}$ Department of Electrical and Electronics Engineering, Shiraz University of Technology, Shiraz, Iran \\ ${ }^{3}$ Department of Electrical Engineering, University of Calgary, Calgary Alberta Canada \\ * Corresponding author's Email: adanbax@gmail.com
}

\begin{abstract}
There are numerous optimization problems in various sciences that need to be solved using the appropriate technique. One of the most widely used techniques for solving optimization problems are population-based optimization algorithms. The innovation and contribution of this paper is to design a new optimizer called Mixed Leader Based Optimizer (MLBO) to solve optimization problems. The main idea in the proposed MLBO is to create a new member as a leader by mixing the best population member and a random member to guide the algorithm population. The main advantage and feature of the proposed MLBO is that it has no control parameters and therefore no need to adjust the parameter. The proposed MLBO algorithm is mathematically formulated to implement in solving various optimization problems. The capability of the proposed optimizer in optimizing and providing appropriate solutions has been tested on a set of twenty-three standard objective functions. These objective functions are selected from three different types including unimodal, high-dimensional multimodal, and fixed-dimensional multimodal in order to analyze different aspects of optimization algorithms. Also, in order to analyze the obtained optimization results, the performance of the MLBO is compared with eight other well-known algorithms including Particle Swarm Optimization (PSO), Genetic Algorithm (GA), Teaching Learning-Based Optimization (TLBO), Gravitational Search Algorithm (GSA), Gray Wolf Optimizer (GWO), Emperor Penguin Optimizer (EPO), Shell Game Optimization (SGO), and Hide Objects Game Optimization (HOGO). The obtained optimization results from the MLBO show the proper performance of the proposed algorithm in solving various optimization problems. On the other hand, comparing the performance of the MLBO with the other eight optimization algorithms indicates the superiority of the proposed optimizer over the compared algorithms.
\end{abstract}

Keywords: Optimization, Population-based method, Optimization problems, Best member, Mixed leader.

\section{Introduction}

Optimization is the science of achieving the best solution among the available solutions to an optimization problem based on the limitations of that problem. Optimization problem solving methods are generally classified into two groups: deterministic methods and stochastic methods [1].

Deterministic methods include two categories of gradient-based and non-gradient-based methods. The most important disadvantage of gradient-based methods is the inability to solve complex highdimensional objective functions as well as nonderivative objective functions. Although non- gradient-based methods can be used in nonderivative environments, these methods are highly dependent on the initial conditions and their solution time increases due to the complexity and dimensions of the objective function [2].

On the other hand, population-based optimization algorithms as a stochastic method, without the need to derivative information of objective functions and based on random search of problem-solving space, are able to provide appropriate solutions to optimization problems [3]. Population-based optimization algorithms are methods that first produce a certain number of random solutions (equal to number of population members) to the 
optimization problem, then in a repetitive process and based on the steps and process of the algorithms improve those solutions [4]. Population-based optimization algorithms are inspired by various natural phenomena, physical laws, swarm behaviors, scientific phenomena, rules of the games, and any other process or idea that has an evolving process.

Each optimization problem has a basic solution called global optimal solution. The important point is that the solutions obtained using population-based optimization algorithms are not necessarily equal to the global optimal solution. For this reason, the solutions obtained from the population-based optimization algorithms are called quasi-optimal solutions [5]. Appropriate quasi-optimal solutions, even if not equal to the global optimal, must be very close to it. In this regard, many population-based optimization algorithms are developed by researchers with the aim of more appropriate quasi-optimal solutions and closer to the global optimal. In this regard, optimization algorithms have been applied by scientists in various fields such as operation of the electricity network [6, 7], energy [8, 9], energy commitment [10,11], protection [12], placement of electrical equipment [13-15], and energy carriers [16, 17] to achieve the optimal solution.

The main innovation and contribution of this paper is designing a new optimizer called Mixed Leader Based Optimizer (MLBO) for solving optimization problems in various fields of science. The main idea in the proposed MLBO is to create a new member as a leader by mixing the best population member and a random member to guide and update the algorithm population. The main advantage and feature of the proposed MLBO is that it has no control parameters and therefore no need to adjust the parameter. The various steps of the proposed MLBO algorithm are explained and then mathematically formulated to implement in solving various optimization problems. The performance of the proposed optimizer in optimizing and providing appropriate solutions are evaluated on a set of twentythree standard objective functions. Also, in order to analyze the obtained optimization results, the performance of the MLBO is compared with eight other well-known algorithms.

The rest of this paper is organized that Section 2 provides an overview of optimization algorithms. The proposed MLBO algorithm is introduced in Section 3. Simulation studies are presented in Section 4. Finally, in Section 5, conclusions and several suggestions for future studies are presented.

\section{Background}

Population-based optimization algorithms from the perspective of the main design idea can be divided into four groups: swarm based, physics-based, evolutionary-based, and game-based optimization algorithms.

Swarm-based optimization algorithms are developed inspired by natural phenomena and swarm behaviors of animals, birds, plants, aquatic animals and other living organisms. One of the oldest and most famous algorithms in this group is the Particle Swarm Optimization (PSO). PSO is inspired by the natural behavior and swarm movement of birds [18]. The main disadvantage of water is that it has several control parameters that must be adjusted properly. The natural behavior of wolves during hunting are used in the design of the Grey Wolf Optimizer (GWO) [19]. Disadvantages of GWO are low solving precision, slow convergence, and bad local searching ability. Simulation of teaching and learning process between students and teacher are used in designing the Teaching-Learning-Based Optimization (TLBO) [20]. Among the disadvantages of TLBO is that consumes lot of memory space, involves lot of iterations so is a time-consuming method. Emperor Penguin Optimizer (EPO) is designed based on simulation of the huddling behavior of emperor penguins [21]. The main disadvantage of EPO is that in order to find the optimal solutions, it is necessary to consider the population size and repetition large enough to search the problem space well, so EPO is a time-consuming method.

Physics-based optimization algorithms are developed based on simulations of various laws and phenomena of physics. Gravitational Search algorithm (GSA) is one of the algorithms in this group, which is designed based on the simulation of gravitational force between different objects that are located at certain distances from each other [22]. Disadvantages of GSA include high computations, large memory space, and fall into local optimal solution.

Evolutionary-based optimization algorithms are developed with inspiration and simulation from the genetics science, the theory of evolution and the process of reproduction. Genetic Algorithm (GA), which is one of the most widely used and oldest optimization algorithms, belongs to this group. GA is designed based on the simulation of the reproductive process, taking into Darwin's theory of evolution in three stages of selection, crossover, and mutation [23]. Disadvantages of GA can be mentioned as follows. GA implementation is still an art. GA requires less information about the problem, but designing an objective function and getting the representation and 
operators right can be difficult. GA is computationally expensive meaning time-consuming.

Game-based optimization algorithms are developed based on simulating the behavior of players and referees in various individual and group games. The behavior of the players and the referee in finding the hidden object in the hide object game is applied in the design of the Hide Objects Game Optimization (HOGO) [24]. HOGO offers more suitable quasi-optimal solutions than similar optimization algorithms, but in solving some optimization problems, quasi-optimal solutions need to be provided closer to the global optimal. The simulation of find the ball hidden under one of the three shells, which should be guessed by players is used in the design of the Shell Game Optimization (SGO) [25]. Although SGO has a good performance in optimizing various optimization problems, it has a high computational volume.

Although criteria such as "time-consuming", "high computational volume", and "large memory space" are discussed about optimization algorithms, but the most important indicator in comparing the performance and ability of optimization algorithms is the value of the objective function. In fact, in comparing the performance of several optimization algorithms on the optimization of an optimization problem, the algorithm that offers the most suitable quasi-optimal solution has a higher capability. Another important point about optimization algorithms is that one algorithm may perform best in optimizing one optimization problem but failing to optimize another optimization problem. This is due to the fact that no optimization algorithm can be considered the best optimizer for all optimization problems. Therefore, in order to compare the performance of optimization algorithms with each other in solving different optimization problems, they must first be implemented on the optimization problem, then based on the values obtained for the objective function of the problem, the algorithm that proposes the best solution is determined.

\section{Mixed Leader Based Optimizer}

In this section, the theory and various steps of the proposed Mixed Leader Based Optimizer (MLBO) are described, then its mathematical formulation is presented to use in solving various optimization problems. MLBO is a population-based optimization algorithm that initially generates a certain number of feasible solutions (as an algorithm population) at random for an optimization problem. MLBO then updates the proposed solutions in an iterative process. At the end of the repetitions, MLBO provides a suitable quasi-optimal solution. The main idea in updating and guiding the algorithm population is to use a new member as the population leader, which is created by mixing the best member of the population with a random member.

The algorithm population of the MLBO is defined as a matrix called the population matrix using Eq. (1).

$$
X=\left[\begin{array}{c}
X_{1} \\
\vdots \\
X_{i} \\
\vdots \\
X_{N}
\end{array}\right]_{N \times m}=\left[\begin{array}{ccccc}
x_{1,1} & \cdots & x_{1, d} & \cdots & x_{1, m} \\
\vdots & \ddots & \vdots & \ddots & \vdots \\
x_{i, 1} & \cdots & x_{i, d} & \cdots & x_{i, m} \\
\vdots & \ddots & \vdots & \ddots & \vdots \\
x_{N, 1} & \cdots & x_{N, d} & \cdots & x_{N, m}
\end{array}\right]_{N \times m}
$$

Here, $X$ is the algorithm population, $X_{i}$ is the $i^{\prime}$ th population member, $N$ is the number of algorithm population members, $m$ is the number of problem variables, $x_{i, d}$ is the value of $d$ 'th problem variable which suggested by $i$ th population member.

Each member of the population matrix as a solution to the problem sets values for the problem variables. Therefore, according to the values specified by each member for the problem variables, a value is obtained for the objective function of the problem. Therefore, these values of the objective function are represented as a vector using Eq. (2).

$$
F=\left[\begin{array}{c}
F_{1} \\
\vdots \\
F_{i} \\
\vdots \\
F_{N}
\end{array}\right]_{N \times 1}=\left[\begin{array}{c}
F_{1}\left(X_{1}\right) \\
\vdots \\
F_{i}\left(X_{i}\right) \\
\vdots \\
F_{N}\left(X_{N}\right)
\end{array}\right]_{N \times 1}
$$

Here, $F$ is the objective function vector and $F_{i}$ is the objective function value for $i$ 'th population member.

In each iteration of the MLBO, based on the comparison of the values of the objective function, the population member that provides the best value for the objective function is considered the best population member. The random member is generated as a feasible solution using Eq. (3).

$$
x_{d}^{R}=x_{d}^{l o}+r \times\left(x_{d}^{h i}-x_{d}^{l o}\right)
$$

Here, $x_{d}^{R}$ is the $d$ 'th dimension of random member, $x_{d}^{l o}$ is the lower bound of $d$ 'th problem variable, $x_{d}^{h i}$ is the upper bound of $d$ 'th problem variable, and $r$ is a random number in [0 $\left.\begin{array}{ll}0 & 1\end{array}\right]$ interval.

In each iteration of the MLBO, after updating the best member and generating a random member, the mixed leader of population is updated based on these two members using Eqs. (4) and (5). 


$$
\begin{gathered}
x_{d}^{M L}=\left(1-I_{M}\right) \times x_{d}^{\text {best }}+I_{M} \times x_{d}^{R} \\
I_{M}=\left\{\begin{aligned}
\left(1-\frac{t}{T}\right), & t<\frac{T}{2} \\
0, & \text { else }
\end{aligned}\right.
\end{gathered}
$$

Here, $x_{d}^{M L}$ is the $d^{\prime}$ th dimension of mixed leader, $I_{M}$ is the mixed index, $x_{d}^{b e s t}$ is the $d^{\prime}$ 'th dimension of best member, $t$ is the iteration counter, and $T$ is the maximum number of iterations.

The population matrix update in the MLBO is simulated based on the mixed leader of population using Eqs. (6) to (8).

$$
\begin{gathered}
d x_{i, d}=\left\{\begin{array}{l}
r \times\left(x_{d}^{M L}-x_{i, d}\right), F_{M L}<F_{i} \\
r \times\left(x_{i, d}-x_{d}^{M L}\right), \text { else }
\end{array}\right. \\
x_{i, d}^{\text {new }}=x_{i, d}+d x_{i, d} \\
X_{i}=\left\{\begin{array}{c}
X_{i}^{\text {new }}, F_{i}^{\text {new }}<F_{i} \\
X_{i}, \text { else }
\end{array}\right.
\end{gathered}
$$

Here, $d x_{i, d}$ is the value of displacement for $i$ th population member in $d^{\prime}$ th dimension, $F_{M L}$ is the objective function value of mixed leader, $x_{i, d}^{\text {new }}$ is the $d$ 'th dimension of new suggested position for $i^{\prime}$ th population member, and $F_{i}^{\text {new }}$ is the objective function value for new suggested position of $i$ th population member.

The steps of updating the population matrix are continued in an iterative-based process using Eqs. (3) to (8). After completing the iterations of the algorithm, the proposed MLBO makes available the obtained best quasi-optimal solution. The flowchart of the implementation steps of the proposed MLBO for use in solving optimization problems is presented in Fig. 1.

\section{Simulation study and discussion}

In this section, the capability of the proposed MLBO in solving various optimization problems and providing appropriate quasi-optimal solutions is analysed. To achieve this purpose, the proposed optimizer has been tested on a set of twenty-three standard objective functions of three types: unimodal functions, high-dimensional multi-model functions, and fixed-dimensional multi-model functions.

In order to analyse the results and evaluate the ability of the proposed MLBO in optimization, the performance of the MLBO is compared with eight other well-known optimization algorithms including Particle Swarm Optimization (PSO), Genetic Algorithm (GA), Teaching Learning-Based

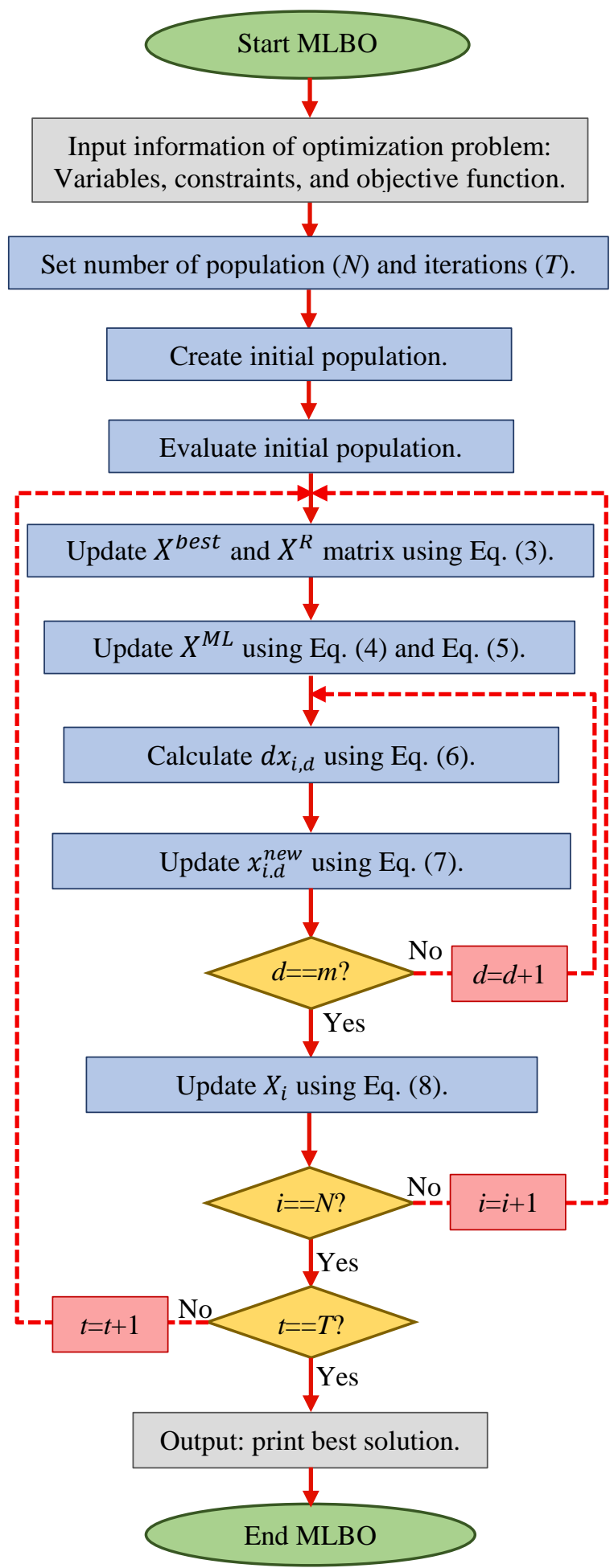

Figure. 1 Flowchart of MLBO

Optimization (TLBO), Gravitational Search Algorithm (GSA), Gray Wolf Optimizer (GWO), Emperor Penguin Optimizer (EPO), Shell Game Optimization (SGO), and Hide Objects Game Optimization (HOGO). The performance results of the MLBO and eight other algorithms are reported using the average of the obtained best quasi-optimal solutions (ave) and the standard deviation of the obtained best quasi-optimal solutions obtained (std). 
Objective functions F1 to F7 are selected to evaluate the performance of optimization algorithms in unimodal objective functions type problems. The results of the implementation of the MLBO and eight compared optimization algorithms are reported in Table 1. The obtained results from the MLBO and its comparison with the performance of the other eight optimization algorithms show that the MLBO has a high ability to solve unimodal optimization problems.

Objective functions F8 to F13 are selected to evaluate the performance of optimization algorithms in providing quasi-optimal solutions to highdimensional multi modal optimization problems. The results of optimization of this type of objective functions using the MLBO and the eight compared optimization algorithms are reported in Table 2. Analyzing and comparing the performance results of optimization algorithms against the proposed algorithm, shows the appropriate ability of the MLBO to be used in solving high-dimensional multimodal optimization problems.

Objective functions F14 to F23 are selected to test the power of optimization algorithms in solving fixed-dimensional multi-modal optimization problems. The performance evaluation results of the MLBO and eight other optimization algorithms in providing quasi-optimal solutions are reported in Table 3. The optimization results which obtained from the implementation of optimization algorithms, indicate the proper performance of the MLBO in solving fixed-dimensional multi-modal optimization problems.

\section{Conclusion and future works}

Population-based optimization algorithms are one of the efficient tools in solving various optimization problems in all sciences. In this paper, a new population-based optimization algorithm called Mixed Leader Based Optimizer (MLBO) that can be used to optimize various optimization problems has been introduced. The main idea of the MLBO was to effectively update the population using a new member called the leader, which has been created by mixing the best member of the population with a random member. The theory and different stages of implementation of the MLBO have been described and then mathematically formulated for use in solving optimization problems.

The capability of the MLBO in solving and presenting quasi-optimal solutions suitable for optimization problems has been tested on a set of twenty-three standard objective functions including unimodal functions, high-dimensional multi-model functions, and fixed-dimensional multi-model
Table 1. Results of MLBO and other algorithms for Unimodal test functions

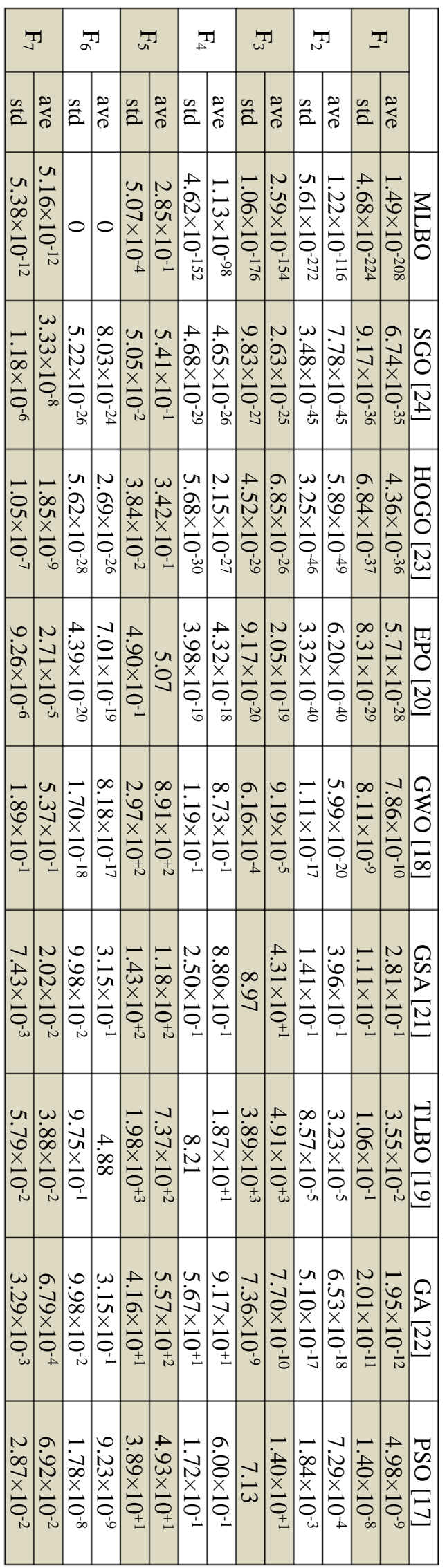


Table 2. Results of MBMBO and other algorithms for high-dimensional Multimodal test functions

\begin{tabular}{|c|c|c|c|c|c|c|c|c|c|c|c|c|}
\hline I & & $\frac{T}{N}$ & & $=$ & & $\frac{T}{0}$ & & & & ]$_{0}^{7}$ & & \\
\hline 2 & 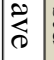 & $\frac{n}{\tilde{a}}$ & 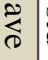 & & 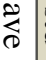 & & $\bar{c}$ & $\vec{\Sigma}$ & 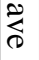 & $\underline{a}$ & & \\
\hline$\stackrel{\circ}{\circ}$ & 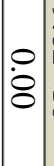 & $\begin{array}{l}0 \\
\hat{\tilde{N}} \\
x \\
0 \\
\vdots \\
\dot{0}\end{array}$ & 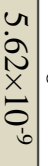 & 0 & & 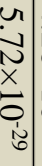 & 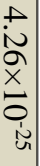 & 0 & 0 & $\begin{array}{l}\vec{u} \\
\vec{x} \\
\underline{x} \\
0 \\
\frac{1}{f}\end{array}$ & $\begin{array}{l}1 \\
\dot{x} \\
0 \\
\\
+\end{array}$ & \\
\hline$\stackrel{\circ}{\circ}$ & $:$ & $\begin{array}{l}\stackrel{+}{ \pm} \\
\overrightarrow{\vec{x}} \\
\vec{\sigma} \\
\dot{+}\end{array}$ & $\begin{array}{l}a \\
\dot{\omega} \\
\dot{x} \\
\sigma \\
0 \\
\dot{\omega}\end{array}$ & 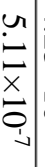 & 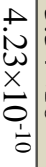 & $\begin{array}{c}\omega \\
w \\
\vec{x} \\
\underline{x} \\
\hat{0} \\
\frac{1}{\infty}\end{array}$ & 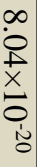 & 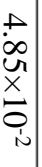 & $\begin{array}{l}\infty \\
\dot{\vec{a}} \\
\bar{x} \\
\dot{\vec{t}} \\
\dot{+}\end{array}$ & 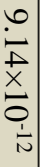 & $\begin{array}{l}1 \\
\dot{N} \\
x \\
\dot{x} \\
\dot{+}\end{array}$ & $\frac{\Omega}{\Omega}$ \\
\hline $\begin{array}{l}0 \\
8 \\
8\end{array}$ & $:$ & $\begin{array}{l}\omega \\
0 \\
a \\
x \\
0 \\
0 \\
+\end{array}$ & $\begin{array}{l}\dot{0} \\
\infty \\
\dot{x} \\
x \\
0 \\
\dot{u}\end{array}$ & 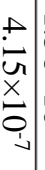 & $\begin{array}{l}\overline{\dot{y}} \\
\hat{x} \\
\vdots \\
\vdots \\
\vdots\end{array}$ & 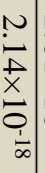 & 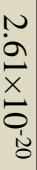 & 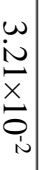 & $\begin{array}{l}u \\
\hat{N} \\
x \\
x \\
0 \\
+\end{array}$ & $\begin{array}{l}\infty \\
\dot{N} \\
\tilde{N} \\
x \\
0 \\
\frac{1}{n} \\
\end{array}$ & 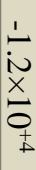 & $\begin{array}{l}\frac{1}{0} \\
0 \\
0 \\
\mathcal{N} \\
\mathcal{\omega}\end{array}$ \\
\hline 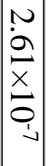 & 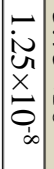 & $\begin{array}{l}\omega \\
\dot{v} \\
\\
x \\
0 \\
\dot{\omega}\end{array}$ & $\begin{array}{l}u \\
0 \\
0 \\
0 \\
x \\
0 \\
\dot{\omega} \\
\end{array}$ & 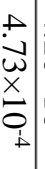 & 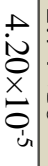 & 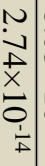 & $\begin{array}{l}\infty \\
0 \\
\dot{w} \\
x \\
0 \\
\frac{1}{\alpha}\end{array}$ & 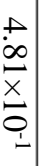 & 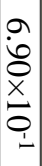 & $\begin{array}{l}u \\
\dot{0} \\
\tilde{x} \\
x \\
0 \\
\pm\end{array}$ & 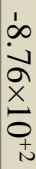 & $\frac{\pi}{0}$ \\
\hline 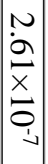 & $\begin{array}{c}0 \\
\dot{0} \\
\dot{\vec{x}} \\
\underline{0} \\
\dot{\infty} \\
\dot{\infty}\end{array}$ & $\begin{array}{c}a \\
\dot{u} \\
\dot{x} \\
x \\
0 \\
\vdots \\
+\end{array}$ & $\begin{array}{c}- \\
\dot{w} \\
\dot{x} \\
\dot{x} \\
\dot{0} \\
\dot{u}\end{array}$ & $\begin{array}{c}-\vec{w} \\
\dot{\vec{x}} \\
\underline{\sigma} \\
0 \\
+\end{array}$ & 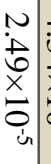 & 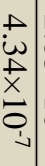 & 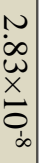 & $\begin{array}{l}w \\
\dot{\sigma} \\
\vec{x} \\
\vec{\sigma} \\
\dot{\underline{t}}\end{array}$ & $\begin{array}{l}+ \\
\infty \\
u_{1} \\
x \\
0 \\
\dot{N}\end{array}$ & $\begin{array}{l}\omega \\
\dot{0} \\
\dot{x} \\
\dot{x} \\
\dot{0} \\
+\end{array}$ & $\begin{array}{l}\dot{+} \\
\dot{0} \\
\dot{x} \\
x \\
\dot{0} \\
\dot{ \pm}\end{array}$ & $\underset{\infty}{\infty}$ \\
\hline 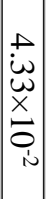 & 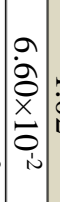 & & $\overline{\mathrm{N}}$ & 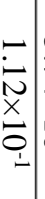 & 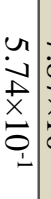 & 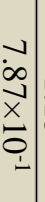 & $\dot{\bar{u}}$ & 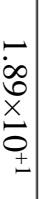 & $\frac{\overrightarrow{0}}{\stackrel{0}{x}}$ & $\begin{array}{l}0 \\
\dot{0} \\
\dot{x} \\
0 \\
\dot{x} \\
0 \\
0 \\
+\end{array}$ & 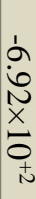 & \\
\hline 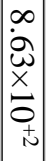 & 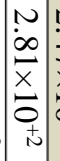 & 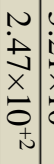 & 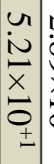 & $\begin{array}{l}N \\
\infty \\
0 \\
x \\
x \\
0 \\
\underline{\Delta}\end{array}$ & 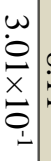 & $\stackrel{\infty}{=}$ & 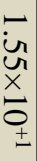 & 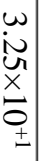 & $\begin{array}{l}\hat{N} \\
\tilde{N} \\
x \\
0 \\
\vdots \\
\pm\end{array}$ & $\begin{array}{l}N \\
\infty \\
\omega \\
x \\
0 \\
\vdots \\
\pm\end{array}$ & 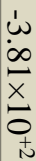 & שֶ \\
\hline 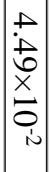 & $\begin{array}{l}a \\
\dot{\omega} \\
\dot{0} \\
x \\
\dot{x} \\
\dot{0} \\
\dot{i}\end{array}$ & $\left.\begin{array}{c}\vec{\infty} \\
\infty \\
\infty \\
x \\
\vdots \\
\dot{0} \\
\dot{\sim}\end{array}\right]$ & 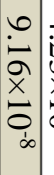 & 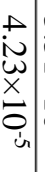 & $\begin{array}{l}\omega \\
\omega \\
\omega \\
x \\
o \\
\dot{\alpha}\end{array}$ & $\begin{array}{l}\overrightarrow{\bar{\sigma}} \\
\bar{x} \\
\overline{0} \\
\dot{0}\end{array}$ & $\begin{array}{l}u \\
\omega \\
\underline{x} \\
\underline{x} \\
0 \\
\underline{\theta} \\
=\end{array}$ & 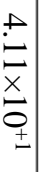 & 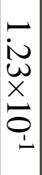 & 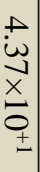 & 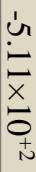 & $\frac{\Omega}{N}$ \\
\hline 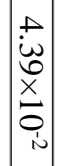 & $\mid \begin{array}{l}a \\
\dot{N} \\
\hat{x} \\
x \\
0 \\
\vdots \\
i\end{array}$ & 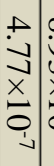 & $\begin{array}{l}\infty \\
\dot{0} \\
\omega \\
x \\
0 \\
\dot{\infty} \\
\end{array}$ & $\begin{array}{l} \pm \\
\dot{\bar{x}} \\
\underline{\underline{x}}\end{array}$ & $\begin{array}{l}\omega \\
\vdots \\
\dot{+} \\
\dot{x} \\
\vdots \\
\vdots \\
\vdots\end{array}$ & $\begin{array}{c}\dot{0} \\
\infty \\
x \\
\dot{x} \\
\vdots \\
\frac{1}{\sigma}\end{array}$ & 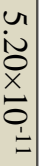 & $\begin{array}{l}\stackrel{+}{0} \\
\stackrel{x}{x}\end{array}$ & 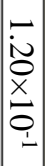 & $\begin{array}{l}\dot{1} \\
\underset{\infty}{\infty} \\
x \\
x \\
0 \\
\pm \\
\end{array}$ & 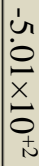 & 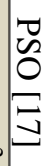 \\
\hline
\end{tabular}

Table 3. Results of MLBO and other algorithms for fixeddimensional Multimodal test functions

\begin{tabular}{|c|c|c|c|c|c|c|c|c|c|}
\hline W & ${ }_{N}^{T}$ & $\stackrel{N}{\mathbb{N}}$ & గ్ర & $\frac{\pi}{6}$ & $\underset{\infty}{T}$ & $\mathbb{T}$ & $\frac{\pi}{2}$ & $\frac{\pi}{4}$ & $T_{F}^{T}$ \\
\hline & 18 & & & & & & & & $\approx$ \\
\hline
\end{tabular}

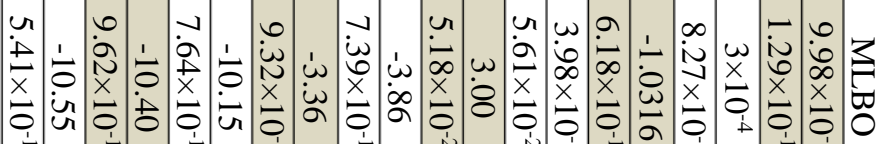

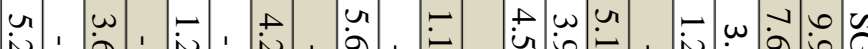

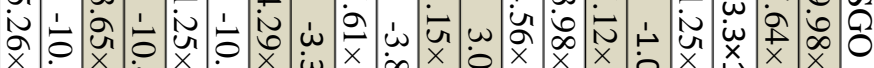

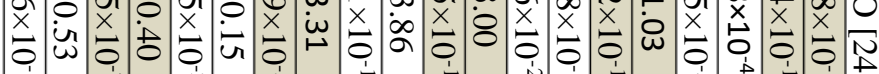

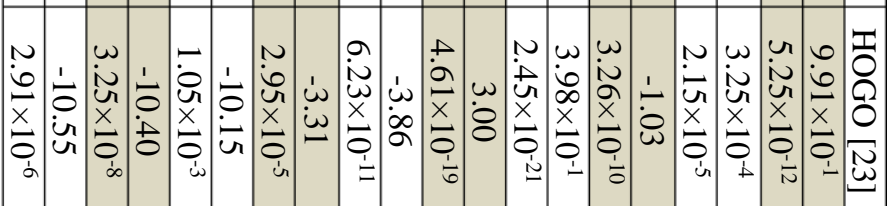

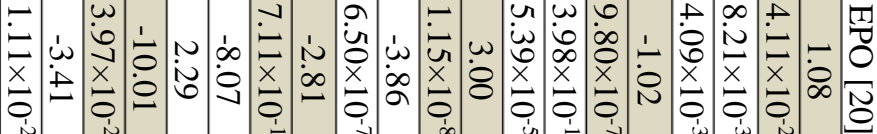

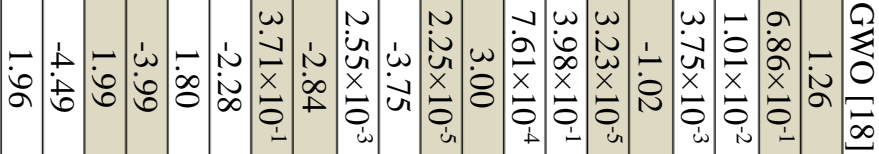

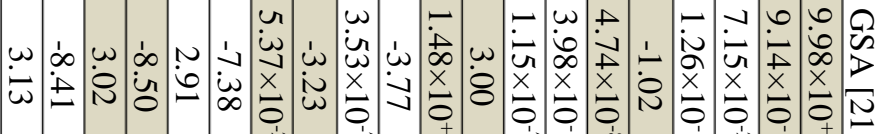

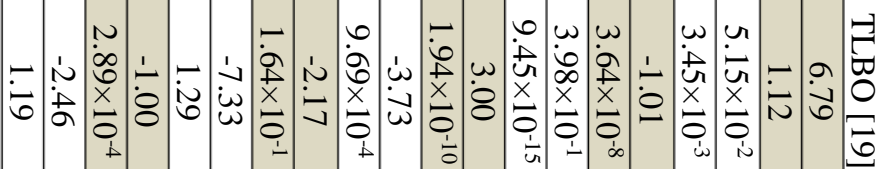

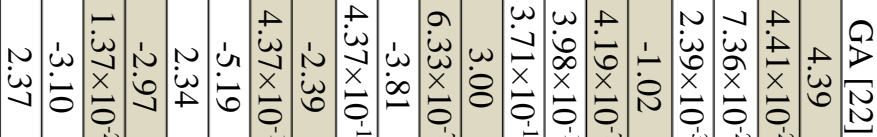

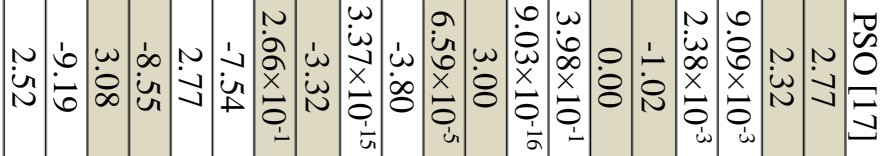

functions. The results of optimization of objective functions indicated the high performance of the International Journal of Intelligent Engineering and Systems, Vol.14, No.4, 2021 proposed MLBO in solving various optimization problems. Also, in order to analyze the results, the 
performance of the proposed algorithm was compared with eight other well-known optimization algorithms including Particle Swarm Optimization (PSO), Genetic Algorithm (GA), Teaching LearningBased Optimization (TLBO), Gravitational Search Algorithm (GSA), Gray Wolf Optimizer (GWO), Emperor Penguin Optimizer (EPO), Shell Game Optimization (SGO), and Hide Objects Game Optimization (HOGO).

Analyzing the optimization results obtained from the optimization algorithms and comparing them with the performance of the MLBO showed that the proposed MLBO offers more suitable quasi-optimal solutions and is much more desirable and competitive than the other eight compared optimization algorithms.

The authors suggest some ideas and perspectives for future studies. Design of the binary version as well as multi-objective version of MLBO is an interesting topic for future investigations. Moreover, implementing MLBO on various optimization problems and real-world optimization problems could achieve some significant contributions, as well.

\section{Conflicts of Interest}

The authors declare no conflict of interest.

\section{Author Contributions}

Conceptualization, F.A.Z. and S.A.D.; methodology, M.D.; software, F.A.Z. and M.D.; validation, F.A.Z., O.P.M., and M.D.; formal analysis, O.P.M. and F.A.Z.; investigation, M.D., S.A.D., and O.P.M.; resources, M.D.; data curation, F.A.Z.; writing - original draft preparation, F.A.Z. and S.A.D.; writing - review and editing, O.P.M. and M.D.; visualization, M.D; supervision, M.D.; project administration, F.A.Z. and S.A.D.; funding acquisition, O.P.M.

\section{References}

[1] S. A. Doumari, H. Givi, M. Dehghani, Z. Montazeri, V. Leiva, and J. M. Guerrero, "A New Two-Stage Algorithm for Solving Optimization Problems", Entropy, Vol. 23, No. 4, pp. 491, 2021.

[2] M. Dehghani, Z. Montazeri, A. Dehghani, R. R. Mendoza, H. Samet, J. M. Guerrero, and G. Dhiman, "MLO: Multi Leader Optimizer", International Journal of Intelligent Engineering and Systems, Vol. 13, No. 6, pp. 364-373, 2020.

[3] M. Dehghani, Z. Montazeri, A. Dehghani, H. Samet, C. Sotelo, D. Sotelo, A. Ehsanifar, O. P. Malik, J. M. Guerrero, and G. Dhiman, "DM:
Dehghani Method for modifying optimization algorithms", Applied Sciences, Vol. 10, No. 21, pp. $7683,2020$.

[4] M. Dehghani, Z. Montazeri, G. Dhiman, O. Malik, R. Morales-Menendez, R. A. RamirezMendoza, A. Dehghani, J. M. Guerrero, and L. Parra-Arroyo, "A spring search algorithm applied to engineering optimization problems", Applied Sciences, Vol. 10, No. 18, pp. 6173, 2020.

[5] S. A. Doumari, H. Givi, M. Dehghani, and O. P. Malik, "Ring Toss Game-Based Optimization Algorithm for Solving Various Optimization Problems", International Journal of Intelligent Engineering and Systems, Vol. 14, No. 3, pp. 545-554, 2021.

[6] S. Dehbozorgi, A. Ehsanifar, Z. Montazeri, M. Dehghani, and A. Seifi, "Line Loss Reduction and Voltage Profile Improvement in Radial Distribution Networks Using Battery Energy Storage System", In: Proc. of IEEE 4th International Conference on Knowledge-Based Engineering and Innovation (KBEI), pp.02150219, 2017.

[7] Z. Montazeri and T. Niknam, "Optimal utilization of electrical energy from power plants based on final energy consumption using gravitational search algorithm", Electrical Engineering \& Electromechanics, No. 4, pp. 7073, 2018.

[8] M. Dehghani, Z. Montazeri, and O. P. Malik, "ENERGY COMMITMENT: A PLANNING OF ENERGY CARRIER BASED ON ENERGY CONSUMPTION", Electrical Engineering \& Electromechanics, No. 4, pp. 69-72, 2019.

[9] M. Dehghani, M. Mardaneh, O. P. Malik, J. M. Guerrero, C. Sotelo, D. Sotelo, M. Nazari-Heris, K. Al-Haddad, and R. A. Ramirez-Mendoza, "Genetic Algorithm for Energy Commitment in a Power System Supplied by Multiple Energy Carriers", Sustainability, Vol. 12, No. 23, pp. 10053, 2020.

[10] M. Dehghani, M. Mardaneh, O. P. Malik, J. M. Guerrero, R. Morales-Menendez, R. A. RamirezMendoza, J. Matas, and A. Abusorrah, "Energy Commitment for a Power System Supplied by Multiple Energy Carriers System using Following Optimization Algorithm", Applied Sciences, Vol. 10, No. 17, pp. 5862, 2020.

[11] M. Dehghani, M. Mardaneh, J. M. Guerrero, O. P. Malik, R. A. Ramirez-Mendoza, J. Matas, J. C. Vasquez, and L. Parra-Arroyo, "A new "Doctor and Patient" optimization algorithm: An application to energy commitment problem", Applied Sciences, Vol. 10, No. 17, pp. 5791, 2020. 
[12] A. Ehsanifar, M. Dehghani, and M. Allahbakhshi, "Calculating The Leakage Inductance for Transformer Inter-Turn Fault Detection Using Finite Element Method", In: Proc. of Iranian Conference on Electrical Engineering (ICEE), pp.1372-1377, 2017.

[13] M. Dehghani, Z. Montazeri, and O. Malik, "Optimal sizing and placement of capacitor banks and distributed generation in distribution systems using spring search algorithm", International Journal of Emerging Electric Power Systems, Vol. 21, No. 1, 2020.

[14] M. Dehghani, Z. Montazeri, O. P. Malik, K. AlHaddad, J. M. Guerrero, and G. Dhiman, “A New Methodology Called Dice Game Optimizer for Capacitor Placement in Distribution Systems", Electrical Engineering \& Electromechanics, No. 1, pp. 61-64, 2020.

[15] M. Dehghani, M. Mardaneh, Z. Montazeri, A. Ehsanifar, M. J. Ebadi, and O. M. Grechko, "Spring search algorithm for simultaneous placement of distributed generation and capacitors", Electrical Engineering \& Electromechanics, No. 6, pp. 68-73, 2018.

[16] M. Dehghani, Z. Montazeri, A. Ehsanifar, A. R. Seifi, M. J. Ebadi, and O. M. Grechko, "Planning of energy carriers based on final energy consumption using dynamic programming and particle swarm optimization", Electrical Engineering \& Electromechanics, No. 5, pp. 6271, 2018.

[17] Z. Montazeri and T. Niknam, "Energy Carriers Management Based on Energy Consumption", In: Proc. of IEEE 4th International Conference on Knowledge-Based Engineering and Innovation (KBEI), pp.0539-0543, 2017.

[18] J. Kennedy and R. Eberhart, "Particle swarm optimization", in Proceedings of ICNN'95international conference on neural networks, Vol. 4, ed: IEEE, pp. 1942-1948, 1995.

[19] S. Mirjalili, S. M. Mirjalili, and A. Lewis, "Grey wolf optimizer", Advances in engineering software, Vol. 69, pp. 46-61, 2014.

[20] R. V. Rao, V. J. Savsani, and D. Vakharia, "Teaching-learning-based optimization: a novel method for constrained mechanical design optimization problems", Computer-Aided Design, Vol. 43, No. 3, pp. 303-315, 2011.

[21] G. Dhiman and V. Kumar, "Emperor Penguin Optimizer: A Bio-inspired Algorithm for Engineering Problems", Knowledge-Based Systems, 2018.

[22] E. Rashedi, H. Nezamabadi-Pour, and S. Saryazdi, "GSA: a gravitational search algorithm", Information sciences, Vol. 179, No. 13, pp. 2232-2248, 2009.

[23] A. Bose, T. Biswas, and P. Kuila, "A novel genetic algorithm based scheduling for multicore systems", Smart Innovations in Communication and Computational Sciences, pp. 45-54, 2019.

[24] M. Dehghani, Z. Montazeri, S. Saremi, A. Dehghani, O. P. Malik, K. Al-Haddad, and J. M. Guerrero, "HOGO: Hide Objects Game Optimization", International Journal of Intelligent Engineering and Systems, Vol. 13, No. 4, pp. 216-225, 2020.

[25] D. Mohammad, M. Zeinab, O. P. Malik, H. Givi, and J. M. Guerrero, "Shell Game Optimization: A Novel Game-Based Algorithm", International Journal of Intelligent Engineering and Systems, Vol. 13, No. 3, pp. 246-255, 2020. 